\title{
INVERSE AND INJECTIVITY OF PARALLEL RELATIONS INDUCED BY CELLULAR AUTOMATA
}

\author{
TAKEO YAKU
}

\begin{abstract}
Moore and Myhill showed that Garden-of-Eden theorem [2], [3]. A binary relation over the configurations is said to be "parallel" if it is induced by a cellular (tessellation) automaton. Richardson showed the equivalence between a parallel relation (a nondeterministic parallel map) with the quiescent state to be injective and its inverse to be parallel by the Garden-of-Eden theorem plus compactness of product topology [4]. This paper deals with the inverse and the injectivity when a cellular automaton is given that induces a parallel relation. We give an equivalent condition, concerning only the local map, for the inverse of a parallel relation to be parallel. Furthermore we show an equivalent condition, concerning only the local map, for the injectivity of a parallel map. Consequently, we note that these two conditions are represented by semirecursive predicates.
\end{abstract}

1. Introduction. A cellular automaton-also known as a tessellation structure-is a model of an array of uniformly connected identical finite automata arranged in a $d$-dimensional Euclidean space divided into square cells, where $d$ is called the dimension. The cellular automaton is denoted by $M=\left(V, Z^{d}, X\right.$, $f$ ), where (i) $V$ is the state set of each finite automaton. (ii) $Z$ denoted the integers. (iii) $X$ is a distinct $n$-tuple $\left(x_{1}, x_{2}, \ldots, x_{n}\right)$ from $Z^{d}$, called the neighbourhood index, where $n$ is a positive integer. We will always assume that $x_{1}=\mathbf{0}(\mathbf{0}$ denotes the $p$-tuple of 0 's). $X$ denotes the locations of the finite automata which are connected to each finite automaton. (iv) $f \subseteq V^{n} \times V$ is the state function of each finite automaton called the local relation. We often represent a binary relation $R \subseteq X \times Y$ by a nondeterministic mapping of a subset of $X$ to $2^{Y}$. A totally defined or a deterministic relation denotes a totally defined or a deterministic mapping, respectively.

A configuration is a mapping $Z^{d} \rightarrow V$, which is an assignment of states into the array. Now, the parallel relation $R$ (over the configurations) induced by $M$ is defined as follows: For configurations $c$ and $d$,

$$
(c, d) \in R \Leftrightarrow f\left(c\left(i+x_{1}\right), c\left(i+x_{2}\right), \ldots, c\left(i+x_{n}\right)\right) \ni d(i) \forall i \in Z^{d} .
$$

A binary relation over the configurations is said to be parallel if it is induced by some cellular automaton. A parallel relation $R$ is called a parallel map if $R$ is deterministic, that is, the local relation is deterministic.

Received by the editors January 16, 1974 and, in revised form, May 12, 1974.

AMS (MOS) subject classifications (1970). Primary 68A25, 94A30.

Key words and phrases. Cellular automata, tessellation structures, parallel maps, parallel relations, self-reproducing, Garden-of-Eden.

- American Mathematical Society 1976 
A cellular automaton $M=\left(V, Z^{d}, X, f\right)$ is with the quiscent state if there is a state $v_{q}$ in $V$ such that $f\left(v_{q}, \ldots, v_{q}\right)=\left\{v_{q}\right\}$. The parallel relation $R$ induced by $M$ is with the quiescent state if $M$ is with the quiescent state, i.e., $c_{q} R d$ iff $d=c_{q}$ for the quiescent configuration $c_{q}$. A configuration $c$ is with finite support provided that the set $\left\{i \in Z ; c(i) \neq v_{q}\right\}$ is finite.

$\bar{X}(i)$ denotes the set $\left\{\left(i+x_{1}\right),\left(i+x_{2}\right), \ldots,\left(i+x_{n}\right)\right\}$ and $\bar{X}(A)$ denotes $\cup_{i \in A} \bar{X}(i)$. A pattern is a restriction of a configuration to a finite set. The parallel relation $R_{p}$ over the patterns is defined by: For patterns $p$ and $q,(p, q) \in R_{p}$ iff $\operatorname{dom} p=\bar{X}(\operatorname{dom} q)$ and

$$
f\left(p\left(i+x_{1}\right), p\left(i+x_{2}\right), \ldots, p\left(i+x_{n}\right)\right) \ni q(i) \forall i \in \operatorname{dom} q .
$$

Garden-OF-Eden Theorem (Moore [2] and Myhill [3]). A totally defined parallel map $R$ with the quiescent state is surjective if and only if $R$ is injective restricted to configurations with finite support.

Richardson combined the theorem above and compactness of product topology [4], and gave the following theorem.

THEOREM A (Richardson [4]). A totally defined parallel map $R$ with the quiescent state is injective if and only if the inverse of $R$ is a totally defined parallel map with the quiescent state.

\section{Results.}

Definition. Let $R_{p}$ be a parallel relation over the patterns induced by $M=\left(V, Z^{d}, X, f\right)$. With respect to a finite set $A(0 \in A)$ in $Z^{d}, R_{p}$ is said to be $A$-independent if for any patterns $p, p^{\prime}$ and $q$ such that $\operatorname{dom} q=A, p R_{p} q$, and $p^{\prime} R_{p} q, r R_{p} q$ for the pattern $r$ such that $\operatorname{dom} r=\operatorname{dom} p, r(\mathbf{0})$ $=p^{\prime}(\mathbf{0})$ and $r(i)=p(i)$ for $i \neq \mathbf{0}$.

A set $A$ is said to be sufficiently large with respect to $X$ if $\bar{X}(i) \cap \bar{X}(\mathbf{0})$ $=\varnothing$ or $\bar{X}(i) \cap\left(Z^{d}-\bar{X}(A)\right)=\varnothing$ for any $i$ in $Z^{d}$.

LEMMA 1. Let $R^{-1}$ be the inverse of a totally defined parallel relation $R$ induced by $M=\left(V, Z^{d}, X, f\right)$. If $R^{-1}$ is a parallel relation induced by $M^{\prime}=\left(V, Z^{d}, Y\right.$, $g)$, then $R_{p}$ is $A$-independent for some sufficiently large finite set $A$ in $Z^{d}$.

Proof. Let $A$ be a sufficiently large finite set in $Z^{d}$ such that $\bar{Y}(\mathbf{0})$ $\subseteq A$ and $\mathbf{0} \in A$. Since $R^{-1}$ is parallel, for patterns $p, p^{\prime}$ and $q$, if $\operatorname{dom} q=A$, $p R_{p} q$ and $p^{\prime} R_{p} q$, then $p(\mathbf{0})$ and $p^{\prime}(\mathbf{0})$ are in

$$
g\left(q\left(\mathbf{0}+y_{1}\right), q\left(\mathbf{0}+y_{2}\right), \ldots, q\left(\mathbf{0}+y_{n^{\prime}}\right)\right),
$$

where $Y=\left(y_{1}, y_{2}, \ldots, y_{n^{\prime}}\right)$. Since $f$ is totally defined, then there are patterns $q_{1}$ and $q_{1}^{\prime}$ such that (i) $\bar{Y}(\bar{X}(A))=\operatorname{dom} q_{1}=\operatorname{dom} q_{1}^{\prime}$, (ii) $q_{1}(i)=q_{1}^{\prime}(i)$ $=q(i)$ for $i$ in $A$, and (iii) $q_{1}\left(R^{-1}\right)_{p} p$ and $q_{1}^{\prime}\left(R^{-1}\right)_{p} p^{\prime}$, where $\left(R^{-1}\right)_{p}$ is the parallel relation over the patterns induced by $M^{\prime}$. Thus from $(1), q_{1}\left(R^{-1}\right)_{p} r$ for the pattern $r$ such that $\operatorname{dom} r=\operatorname{dom} p, r(\mathbf{0})=p^{\prime}(\mathbf{0})$ and $r(i)=p(i)$ for $i$ $\neq \mathbf{0}$, since $\bar{Y}(\mathbf{0}) \subseteq A$. Accordingly, $r R_{p} q$, and therefore $R_{p}$ is $A$-independent. Q.E.D.

In order to prove the converse of Lemma 1, we will show in advance the following 
Lemma 2. Let us suppose that $R$ is the parallel relation induced by $M$ $=\left(V, Z^{d}, X, f\right)$. Let $A$ be a finite set in $Z^{d}$ which is sufficiently large with respect to $X$, and $A^{\prime}$ be such as $A^{\prime} \supseteq A$. If $R_{p}$ is $A$-independent, then $R_{p}$ is $A^{\prime}$ independent.

Proof. For any patterns $p_{1}^{\prime}, p_{2}^{\prime}$ and $q^{\prime}$ such that $\operatorname{dom} q^{\prime}=A^{\prime}$, if $p_{1}^{\prime} R_{p} q^{\prime}$ and $p_{2}^{\prime} R_{p} q^{\prime}$, then $p_{1} R_{p} q$ and $p_{2} R_{p} q$ for patterns $p_{1}, p_{2}$ and $q$ such as $p_{1} \subseteq p_{1}^{\prime}, p_{2} \subseteq p_{2}^{\prime}, q \subseteq q^{\prime}, \operatorname{dom} q=A$ and $\operatorname{dom} p_{1}=\operatorname{dom} p_{2}=\bar{X}(A)$. Accordingly $r R_{p} q$ for the pattern $r$ such that $\operatorname{dom} r=\operatorname{dom} p, r(\mathbf{0})$ $=p_{2}(\mathbf{0})$ and $r(i)=p_{1}(i)$ for $i \neq \mathbf{0}$. Let $r^{\prime}$ be a pattern such as (i) dom $r^{\prime}$ $=\bar{X}\left(A^{\prime}\right)$, (ii) $r^{\prime}(\mathbf{0})=p_{2}^{\prime}(\mathbf{0})$, and (iii) $r^{\prime}(i)=p_{1}^{\prime}(i)$ for $i \neq \mathbf{0}$.

We will prove that $r^{\prime} R_{p} q^{\prime}$. Let $r^{\prime \prime}$ be the restriction of $r^{\prime}$ to $\bar{X}(i)$. For $i$ such that $\bar{X}(i) \ni \mathbf{0}$, we obtain $r^{\prime \prime} R_{p} q^{\prime}(i)\left(q^{\prime}(i)=q(i)\right)$, since $r^{\prime \prime}(i)=r(i)$ for $i$ $\in \bar{X}(i), R$ is $A$-independent and $A$ is sufficiently large. For $i$ such as $\bar{X}(i) \ni \mathbf{0}$, it is obvious that $p_{1}^{\prime \prime} R_{p} q^{\prime}(i)$, where $p_{1}^{\prime \prime}$ is the restriction of $p_{1}^{\prime}$ to $\bar{X}(i)$. Q.E.D.

The next theorem does not only show that the inverse of a parallel relation is parallel when $R_{p}$ is $A$-independent, but also shows that we can explicitly give a cellular automaton that induces the inverse, using a cellular automaton defined below.

Let us suppose that $A=\left\{a_{1}, a_{2}, \ldots, a_{m}\right\}\left(a_{1}=\mathbf{0}\right)$ is a finite set in $Z^{d}$ and that $M=\left(V, Z^{d}, X, f\right)$ is a cellular automaton with $X=\left(x_{1}, x_{2}, \ldots, x_{n}\right)$. Let $\left(R_{p}\right)^{-1}$ be the inverse of the parallel relation $R_{p}$ over the patterns induced by $M$. Let $Y=\left(y_{1}, y_{2}, \ldots, y_{n^{\prime}}\right)$ be such that

$$
\bar{X}(A) \subseteq \bigcap_{1 \leqslant i \leqslant n} \bar{Y}\left(x_{i}\right)
$$

and that $n^{\prime}$ is finite. Now, $M(A)=\left(V, Z^{d}, Y, g\right)$ denotes a cellular automaton defined as:

$$
g\left(v_{1}, v_{2}, \ldots, v_{n}\right) \ni v,
$$

if there are patterns $p$ and $q$ such that $p R_{p} q, p(\mathbf{0})=v$ and $p\left(y_{i}\right)=v_{i}$ for any $i\left(1 \leqslant i \leqslant n^{\prime}\right)$ where $\operatorname{dom} p=\bar{X}(\bar{Y}(\mathbf{0}))$ and dom $q=\bar{Y}(\mathbf{0})$.

THEOREM 1. Let $R$ be a parallel relation induced by $M=\left(V, Z^{d}, X, f\right)$ with the inverse $R^{-1}$. Let $A$ be a sufficiently large finite set in $Z^{d}$ with respect to $X$. If the parallel relation $R_{p}$ over the patterns is A-independent, then a cellular automaton $M(A)$ defined in (3) induces $R^{-1}$.

Proof. Let $A$ be such that $A=\left\{a_{1}, a_{2}, \ldots, a_{m}\right\}$. We can assume that $a_{1}=\mathbf{0}$. Assume that $M(A)=\left(V, Z^{d}, Y, g\right), Y=\left(y_{1}, y_{2}, \ldots, y_{n^{\prime}}\right)$ and $S$ is the parallel relation induced by $M(A)$.

We will prove that $d R^{-1} c$ if and only if $d S c$ for configurations $c$ and $d$. Let us assume that $d R^{-1} c$. Let $p$ be the restriction of $c$ to $\bar{X}(\bar{Y}(i))$ and $q$ be the restriction of $d$ to $\bar{Y}(i)$ with respect to any $i$ in $Z^{d}$. Clearly $p R_{p} q$. Hence $p(i) \in g\left(q\left(i+y_{1}\right), q\left(i+y_{2}\right), \ldots, q\left(i+y_{n^{\prime}}\right)\right)$ from (3). Thus $d S c$.

On the other hand, let us assume that $d S c$. We must prove that $d(i)$ $\in f\left(c\left(i+x_{1}\right), c\left(i+x_{2}\right), \ldots, c\left(i+x_{n}\right)\right)$ for any $i$ in $Z^{d}$, where $X=\left(x_{1}\right.$, $\left.x_{2}, \ldots, x_{n}\right)$. Fix $i \in Z^{d}$. 
From (3) there are patterns $p_{1}, p_{2}, \ldots, p_{n}$ for each $1 \leqslant j \leqslant n$ such that

$$
\left\{\begin{array}{l}
\operatorname{dom} p_{j}=\bar{X}\left(\bar{Y}\left(i+x_{j}\right)\right), \\
p_{j}\left(i+x_{j}\right)=c\left(i+x_{j}\right), \\
p_{j} R_{p} q_{j},
\end{array}\right.
$$

where $X=\left(x_{1}, x_{2}, \ldots, x_{n}\right)$ and $q_{j}$ is the restriction of $d$ to $\bar{Y}\left(i+x_{j}\right)$. Let $q_{j}^{\prime}(1 \leqslant j \leqslant n)$ be such that $q_{j}^{\prime}=q_{1} \cap q_{2} \cap \cdots \cap q_{j}$, where mappings $q_{1}$, $q_{2}, \ldots, q_{j}$ are considered as sets.

Let $r_{1}, r_{2}, \ldots, r_{n}$ be the patterns as defined below:

$$
\begin{cases}r_{1}=p_{1}, & \\ \operatorname{dom} r_{j+1}=\operatorname{dom} r_{j} & \cap \operatorname{dom} p_{j+1}, \\ r_{j+1}(x)=r_{j}(x) & \left(x \neq i+x_{j+1}\right), \\ =p_{j+1}(x) & \left(x=i+x_{j+1}\right),\end{cases}
$$

where $1 \leqslant j \leqslant n$. It is clear that $r_{1} R_{p} q_{1}^{\prime}$. We will prove that if $r_{j} R_{p} q_{j}^{\prime}$, then $r_{j+1} R_{p} q_{j+1}^{\prime}$ for $j(1 \leqslant j<n)$.

Assume that $r_{j} R_{p} q_{j}^{\prime}$. Let $r_{j+1}^{\prime}$ be the restriction of $r_{j}$ to dom $r_{j+1}$. We have $r_{j+1}^{\prime} R_{p} q_{j+1}^{\prime}$, while $A$ is sufficiently large and $\left\{i+x_{j+1}+a_{1}, i+x_{j+1}\right.$ $\left.+a_{2}, \ldots, i+x_{j+1}+a_{m}\right\} \subseteq \operatorname{dom} r_{j+1}$ from (2). Thus, from Lemma 2, $r_{j+1} R_{p} q_{j+1}^{\prime}$. Accordingly, $r_{n} R_{p} q_{n}^{\prime}$. Since $q_{n}^{\prime}(i)=d(i)$, the proof is completed. Q.E.D.

THEOREM 2. The inverse of a totally defined parallel relation $R$ induced by $M=\left(V, Z^{d}, X, f\right)$ is parallel if and only if the parallel relation $R_{p}$ over the patterns is $A$-independent for some sufficiently large finite set $A$ with respect to $X$.

The next theorem deals with the injectivity of a parallel map. From Theorems $\mathrm{A}$ and 2

THEOREM 3. A totally defined parallel map $R$ with the quiescent state induced by $M=\left(V, Z^{d}, X, f\right)$ is injective if and only if

(i) the parallel map $R_{p}$ over the patterns is A-independent for some sufficiently large finite set $A$ with respect to $X$, and

(ii) the inverse $R^{-1}$ is totally defined and with the quiescent state.

We note that condition (i) in Theorem 3 is represented by a semirecursive predicate. While, $R^{-1}$ in Theorem 3 is induced by $M(A)$ defined in (3), if $R_{p}$ is $A$-independent. Then:

REMARK. The following are represented by semirecursive predicates:

(i) The inverse of the totally defined parallel relation induced by a given cellular automaton is parallel.

(ii) The totally defined parallel map with the quiescent state induced by a given cellular automaton is injective.

\section{REFERENCES}

1. S. Amoroso and G. Cooper, The Garden-of-Eden theorem for finite configurations, Proc. Amer. Math. Soc. 26 (1970), 158-164. MR 43 \#1760. 
2. E. F. Moore, Machine models of self-reproduction, Proc. Sympos. Appl. Math., vol. 14, Amer. Math. Soc., Providence, R. I., 1962, pp. 17-33.

3. J. Myhill, The converse of Moore's Garden-of-Eden theorem, Proc. Amer. Math. Soc. 14 (1963), 685-686. MR 27 \#5698.

4. D. Richardson, Tessellations with local transformations, J. Comput. System Sci. 6 (1972), 373-388. MR 47 \#8220.

5. T. Yaku, The constructibility of a configuration in a cellular automaton, J. Comput. System Sci. 7 (1973), 481-496. MR 48 \#10724.

6. - Surjectivity of nondeterministic parallel maps induced by nondeterministic cellular automata, J. Comput. System Sci. 12 (1976), 1-5.

Department of Mathematics, Waseda University, Tokyo 160, JaPan

Current address: Department of Mathematical Sciences, Tokai University, Hiratsuka, Kanagawa 259-12, Japan 